\title{
Capacitive Sensors from Low Cost to High Resolution on Printed Circuit Boards
}

\author{
A. Bülau ${ }^{1}$, A. Schwenck ${ }^{1}$, Dr. K.-P. Fritz ${ }^{1}$, Prof.-Dr. H. Kück ${ }^{1}$ \\ ${ }^{1}$ HSG-IMAT, Allmandring 9B, 70569 Stuttgart, Germany, \\ Buelau@hsg-imat.de
}

\begin{abstract}
HSG-IMAT develops capacitive sensors based on printed circuit board technology (pcb) for application specific use. PCB based sensors have a few benefits compared to other sensor technologies, they are flexible in design, layouts can be easily and cost-efficiently adapted to new requirements and standard SMD processes can be used to assemble necessary components and circuits. They are adequate for small and medium-sized quantities and without any special process and they can be manufactured or modified by SMEs themselves. From low cost pressure, wireless readout assembling, torque and rotary position sensors to high resolution 1-axis and 2-axis inclination and position sensors, a wide spectrum of knowledge in capacitive sensor design based on printed circuit board technology is available. With respect to the final application of the sensor, its base capacitance, the required resolution and repetition rate, different integrated circuits and topologies for digitizing the capacitive sensor signal can be chosen. HSG-IMAT also offers a full spectrum of tests for reliability and develops algorithms to compensate the sensors for ambient conditions such as temperature and humidity.
\end{abstract}

Key words: capacitive sensors, pressure sensor, torque \& force sensor, rotary position encoder, inclination \& position sensor

\section{Low cost pressure sensor}

HSG-IMATs capacitive low-cost pressure sensor consists of a pcb with a soldered membrane. Fig. 1 shows a proof of concept study. The pcb carries a first electrode underneath the membrane, which itself is the second electrode and is deformed by a pressure on its top. The membrane acts as a pressure-to-capacitance converter. The sensor cell is measured against a fixed NPO ceramic capacitor.

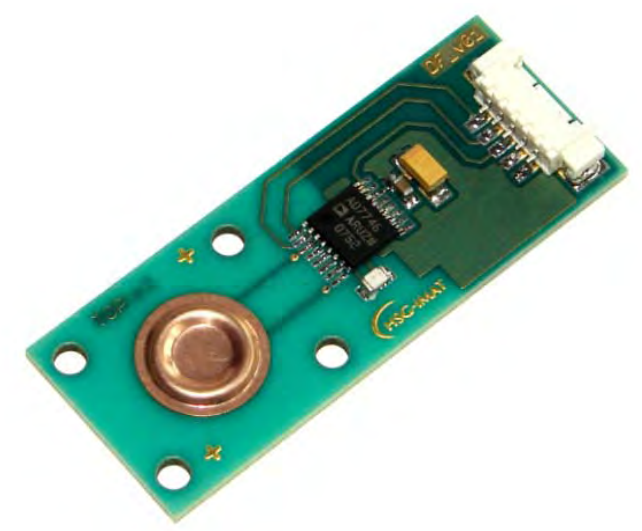

Fig. 1. Low cost capacitive pressure converter membrane on a printed circuit board with the readout circuit.
With this first setup a repeatable accuracy of \pm 20 mbar in a range of 0-7 bar was reached, illustrated in Fig. 2.
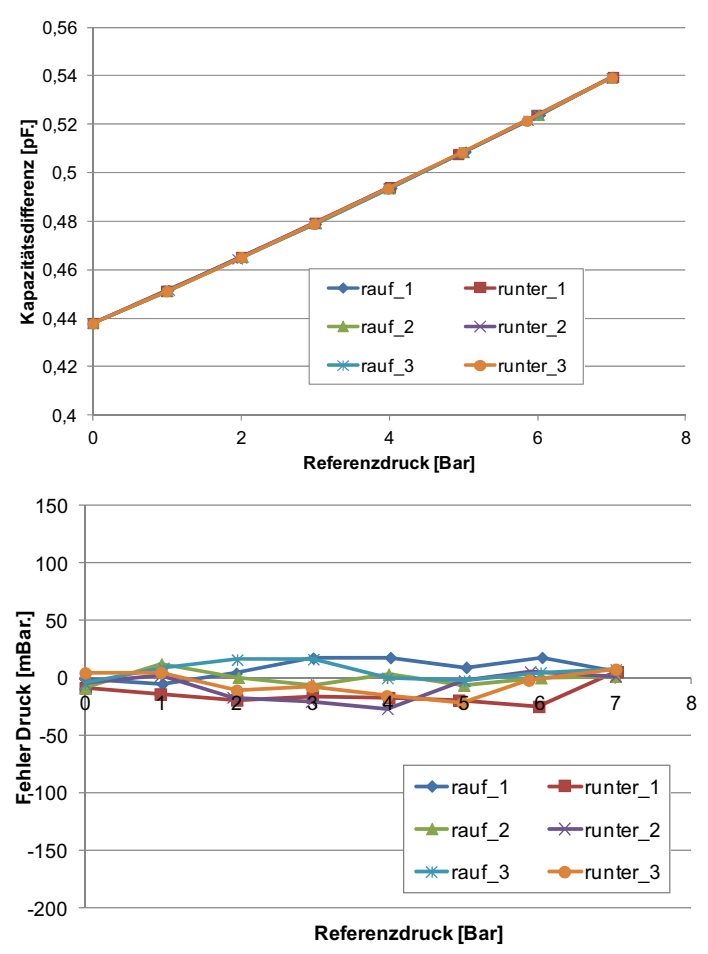

Fig. 2. Sensor characteristics and error graph after second order calibration 
Current developments trace on integrating a fully differential sensor to the pcb to compensate for temperature and humidity influence. This can e.g. be done by replacing the NPO ceramic capacitor by a second membrane without pressure admission to it or by integrating a fully differential structure underneath the membrane. Both solutions are in progress and first results are expected soon.

\section{Low cost torque and rotary position sensor}

New field of application at HSG-IMAT are capacitive low cost torque and rotary position sensors. First bench tests for a torque sensor in flange design with a constructed measurement range of $\pm 100 \mathrm{Nm}$ and a corresponding rotation angle of $\pm 5^{\circ}$ showed promising results of $59.5 \mathrm{mNm}$ in resolution at $45.5 \mathrm{~Hz}$ measurement rate. Fig. 3 shows the proof of concept on a rotary position test bench. It is planned to significantly increase repetition rate by using a new readout circuit, so that for example motor torque can be online monitored.
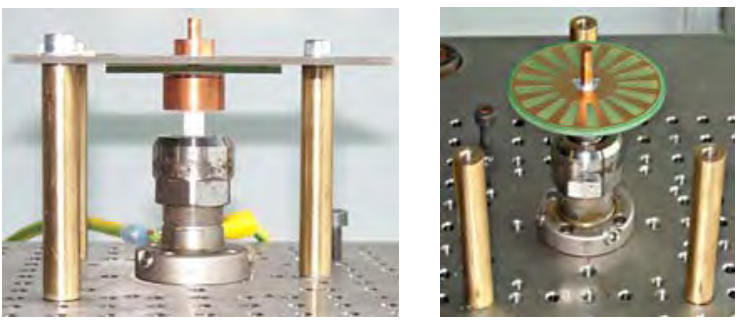

Fig. 3. Low cost capacitive torque sensor on rotary position test bench.

This concept also allows to be used as a capacitive low cost rotary position encoder. In the present case the torque sensor also acts as an encoder with 17 increments (Fig. 4).
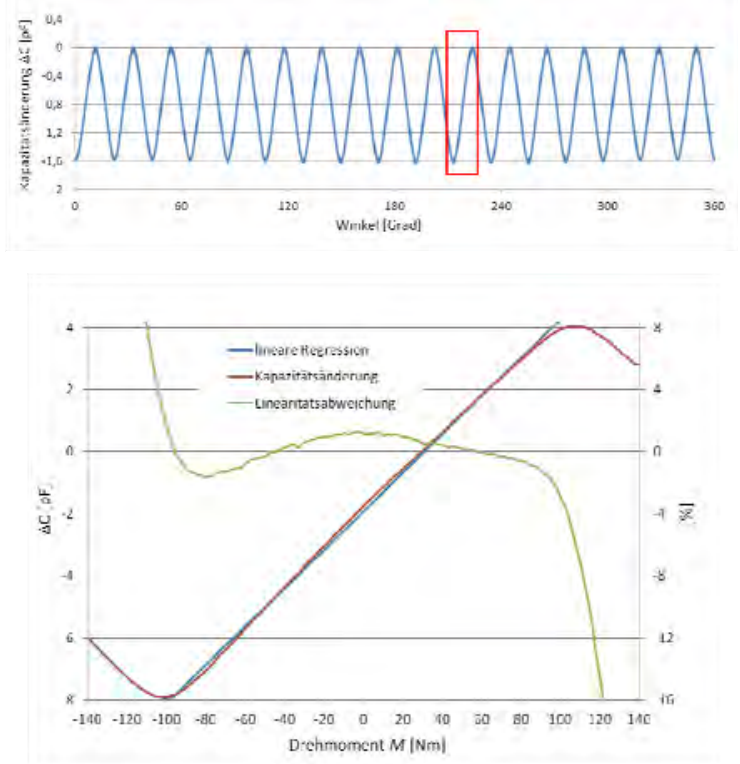

Fig. 4. Sensor characteristic of the torque sensor.

\section{Low cost assembling sensor}

The assembling sensor, developed in collaboration with A. Raymond, is a low cost capacitive sensor on pcb for detecting the assembly condition of fluidic quick connectors in pre-assembled fuel tanks. Therefore the sensor is formed as a resonant circuit with a variable capacitor, shown in Fig. 5.

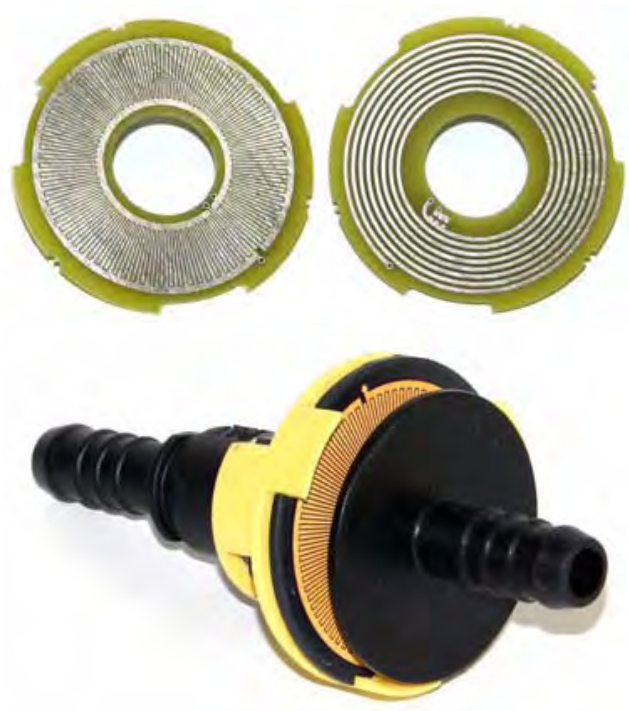

Fig. 5. Low cost capacitive assembling sensor for wireless readout.

The assembly condition is mapped to the resonance frequency of the sensor which is wireless detected by a reader system outside the tank within the frequency range of 7.4 and 8.8 MHz. With a simple mask based pass/fail test the tank with the quick connectors inside can be checked. In Fig. 6 such a pass/fail test is illustrated for one quick connector, but can be extended to multiple quick connectors of different frequencies within the frequency range. This allows the tank manufacturer to deliver fully qualified and online documented fuel tanks for the first time.
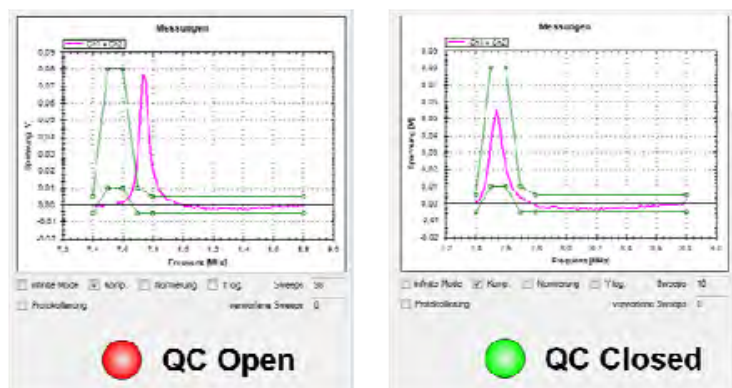

Fig. 6. Mask based pass/fail test of fluidic quick connector with assembling sensor on wireless reader system. 


\section{High resolution inclination sensor}

In 2009 the 1 -axis $360^{\circ}$-inclination sensor developed at HSG-IMAT was successfully transferred into market. This sensor again is completely made of printed circuit boards, stacked to form the sensor element, which is filled with a fluid (Fig. 7).

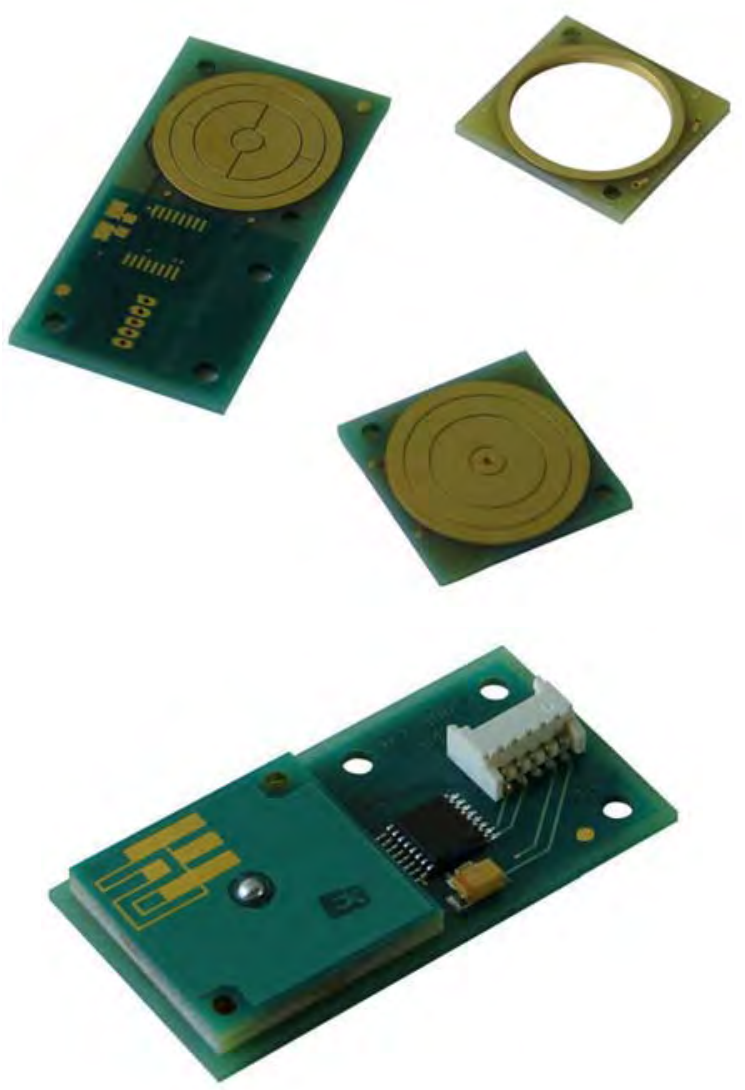

Fig. 7. Capacitive 1-axis high resolution $360^{\circ}$ inclination sensor element.

With its outstanding characteristics the sensor fulfilled all specifications in a benchmark with available silicon sensors and was integrated with a custom design to an OEM laser distance meter (Fig. 8). Beyond this the sensor is also available in a consumer packaged solution by $2 \mathrm{E}$ mechatronic with several interfaces.

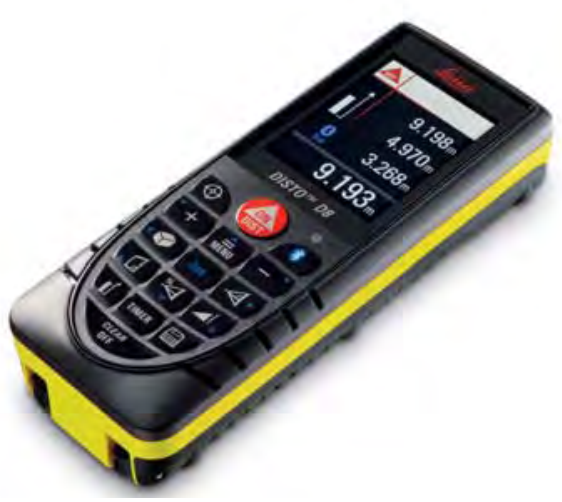

Fig. 8. OEM 1-axis $360^{\circ}$-inclination sensor solution.
HSG-IMATs 2-axes high resolution inclination sensor is a further development based on the 1 -axis sensor. This high resolution sensor consists of a printed circuit board with 4 electrodes in a pie slice arrangement (Fig. 9).

A massive copper dome soldered to the surrounding contact ring acts as a 5th electrode, resulting in two sensitive axes.

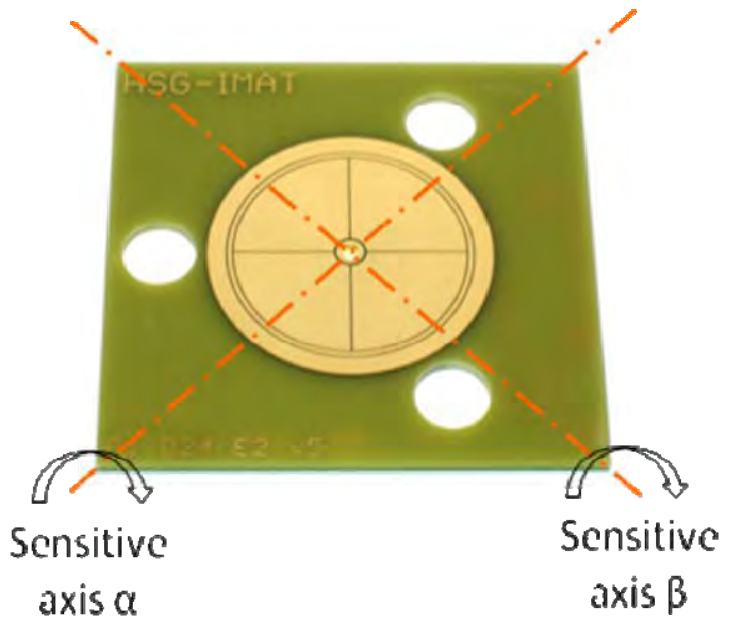

Fig. 9. PCB layout of the capacitive sensor structure.

The inner surface of the dome is of spherical shape turned by an ultra precision machining center in optical surface quality. Further research regarding this surface showed that the dome could also be fabricated on conventional turning center using natural or polycrystalline diamond tools without any disadvantages in sensor performance.

In the space between pcb and dome is a fluid with an air bubble that changes its position as a function of the inclination of the sensor plane. That results in a change of differential capacitance of respectively two diagonal electrodes.

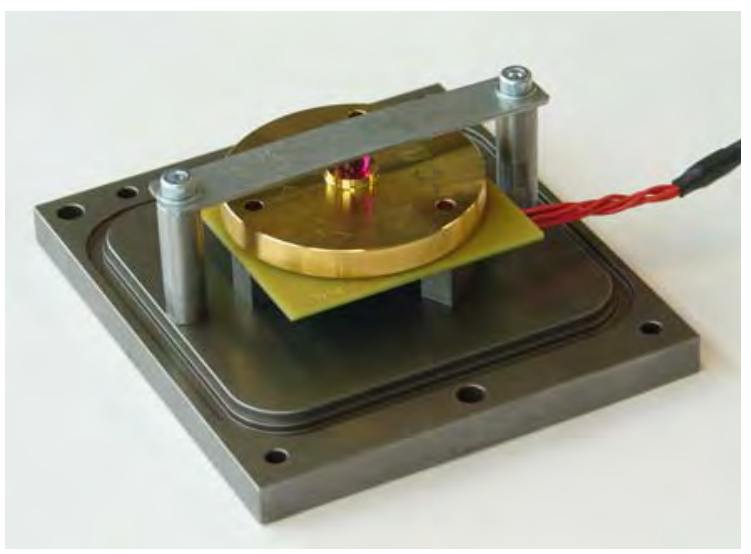

Fig. 10. Capacitive 2-axis high resolution inclination sensor.

The dome itself offers a statically defined threepoint bearing of the sensor and a fourth point 
for fixation via non-conductive sapphire balls assembled to the sensor enclosure (Fig. 10).

With a sensitive range of $\pm 2^{\circ}$ the new sensor allows a high resolution with a standard deviation of around $\pm 5 \mu \mathrm{m} / \mathrm{m}$ at $9 \mathrm{~Hz}$ repetition rate for one axis ( $4.3 \mathrm{~Hz}$ for both axis) which is actual limited by measurement noise (Fig. 11).
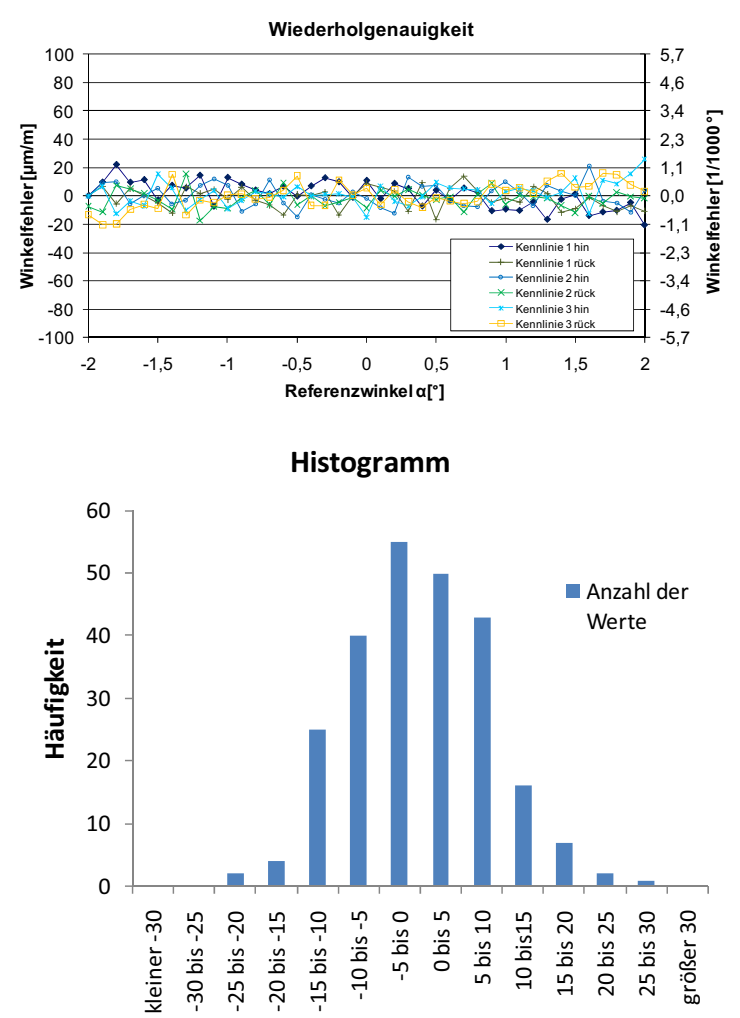

Abweichung $[\mu \mathrm{m} / \mathrm{m}]$

Fig. 11. Repeat accuracy and histogram of one sensor axis.

In the latest version with improved readout circuit, noise was reduced by more than factor 2 so that the standard deviation decreased down to $< \pm 2.5 \mu \mathrm{m} / \mathrm{m}$. HSG-IMAT further works on improving this sensor.

With the careful selection of the fluid the sensor can be set up for special requirements, like high dynamic or sensitivity.

\section{High resolution position sensor}

Within the scope of an industrial project in collaboration with Festo AG \& Co. KG in 2012 HSG-IMAT developed a 1-axis high resolution position sensor based on an electrode arrangement on top of a pcb. The metallic or metalized object to be detected, shorter than $35 \mathrm{~mm}$ and less than $1 \mathrm{~mm}$ in height, is placed $50 \mu \mathrm{m}$ in front of the electrodes and moved in vertical direction (Fig. 12).

The sensor was characterized at HSG-IMAT on a high resolution angle test bench with high resolution reference sensors. The claimed measurement range of $\pm 100 \mu \mathrm{m}$ was considerably exceeded.

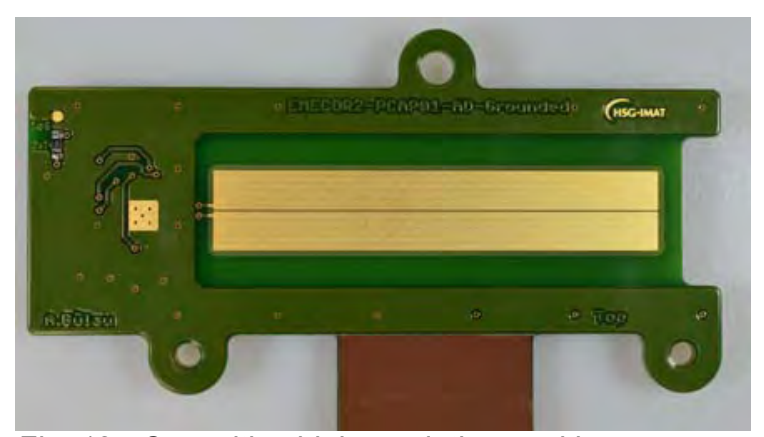

Fig. 12. Capacitive high resolution position sensor.

In a nearly linear range of $\pm 1 \mathrm{~mm}$ resolutions of $< \pm 10 \mathrm{~nm}$ at a repetition rate of $17 \mathrm{~Hz}$ were achieved. This corresponds to a dynamic range of $100 \mathrm{~dB}$. With these parameters the capacitive position sensor expands into a field of dimensions that is normally reserved to optical solutions, such as interferometers.

In a current corporate project the resolution will be further improved and also repetition rate will be investigated.

\section{Miscellaneous}

Next to the presented selection HSG-IMAT developed several other printed circuit boards based sensors, for example force sensor and printed capacitive touch sensors on pcb.

A variety of digitizing circuits allows us to select the optimal circuit solution for each sensor.

With our in house reliability tests HSG-IMAT is able to qualify all developed sensors within given specifications. Therefore we are able to construct and fabricate test benches that can be integrated to the reliability tests.

We evaluate compensation algorithms for the sensors, such as curve fitting, offset correction, temperature and humidity compensation, to achieve the maximum performance of the capacitive sensors.

\section{Conclusion}

In this article we presented a few examples of capacitive sensors fabricated by using standard printed circuit board and packaging technologies. We illustrated the possibility to realize application specific low cost and high resolution sensor solutions with a very flexible technology that is especially suitable for small and medium-sized quantities. The moderate costs of such capacitive sensor developments and fabrication makes this technology adequate for SMEs. 